\title{
Surgical Site Infections in Breast Cancer Patients Undergoing Surgery During the Covid-19 Pandemic. Preliminary Data from an Italian Oncologic Hub.
}

\section{Sonia Cappelli}

IFO: Istituti Fisioterapici Ospedalieri

\section{Fabio Pelle}

IFO: Istituti Fisioterapici Ospedalieri

Marco Clementi

ASL 1 Avezzano Sulmona L'Aquila: Azienda Sanitaria Locale 1 Avezzano Sulmona L'Aquila

\section{Maddalena Barba}

IFO: Istituti Fisioterapici Ospedalieri

\section{Patrizia Vici}

IFO: Istituti Fisioterapici Ospedalieri

Elisabetta Capomolla

IFO: Istituti Fisioterapici Ospedalieri

\section{Laura Pizzuti}

IFO: Istituti Fisioterapici Ospedalieri

\section{Diletta Corallino}

IFO: Istituti Fisioterapici Ospedalieri

Isabella Sperduti

IFO: Istituti Fisioterapici Ospedalieri

\section{Franco Graziano}

IFO: Istituti Fisioterapici Ospedalieri

\section{Loredana Piarulli}

IFO: Istituti Fisioterapici Ospedalieri

\section{Flavia Cavicchi}

IFO: Istituti Fisioterapici Ospedalieri

\section{Domenico Magagnano}

IFO: Istituti Fisioterapici Ospedalieri

\section{Roy De vita}

IFO: Istituti Fisioterapici Ospedalieri

\section{Vittorio Quercia}

IFO: Istituti Fisioterapici Ospedalieri

\section{Marcello Pozzi}

IFO: Istituti Fisioterapici Ospedalieri

Maurizio Costantini 
IFO: Istituti Fisioterapici Ospedalieri

\section{Gennaro Ciliberto}

IFO: Istituti Fisioterapici Ospedalieri

Claudio Botti ( $\sim$ claudio.botti@ifo.gov.it)

IFO: Istituti Fisioterapici Ospedalieri

\section{Research}

Keywords: Covid-19 pandemic, breast cancer, surgical site infections, Center for Disease Control and Prevention.

Posted Date: June 7th, 2021

DOI: https://doi.org/10.21203/rs.3.rs-536238/v1

License: (c) (7) This work is licensed under a Creative Commons Attribution 4.0 International License. Read Full License 


\section{Abstract}

Background: Over the Covid-19 pandemic, the compelling need of containing the virus spread while providing diseased people with adequate assistance has inevitably reflected on treatment priorities, which have been sometimes radically revised according to the intrinsic nature of the institution considered and its role and action within the pandemic context. We report on the experience matured at the Regina Elena National Cancer Institute concerning the effects of the restriction measures adopted during the pandemic on the outcomes of surgical site infections (SSIs) in breast cancer patients (BC-pts) undergoing non-deferrable breast cancer surgery.

Methods: Within the frame of an observational study, we compared evidence from two cohorts, namely, the lockdown cohort (LDC) and non-lockdown cohort (NLDC). Patients from the LDC underwent breast cancer surgery between the $15^{\text {th }}$ March 2020 and the $4^{\text {th }}$ May 2020. Breast surgery was performed by the same team and postsurgical controls were performed regularly for a minimum of 12-months. SSIs were defined according to the criteria established by the Center for Disease Control and Prevention.

Results: The LDC originally included 79 breast cancer patients, of whom 62 provided data to the final analysis. In the LDC, initially 64 breast cancer patients, only 52 were finally considered. No relevant differences emerged between LDC and NLDC by general characteristics. We observed significant evidence of lower SSIs in the subgroups of patients having undergone skin reducing mastectomy compared with the remaining subgroups $(p=0.003)$. The overall number of surgical site infections was $10(8.7 \%)$, and the median time to their occurrence17.1 days (7-42). In the NLDC, 7 (13.5) SSIs were identified, with a median time to occurrence of 18.2 days (7-42). In the LDC, 3 (4.8\%) SSIs occurred, with a median time to occurrence of 14.3 days (7 to 21$)$. None of these patients was in need of re-hospitalization and there were no delays in adjuvant treatment starting.

Conclusions: The restrictive measures issued during the lockdown period seemed to lower the SSI rates in breast cancer patients undergoing non-deferrable breast surgery, although, caution is needed due to the limited sample size.

\section{Background}

Since the $21^{\text {st }}$ of February 2020, the date at the first case of severe acute respiratory syndrome corona virus-2 (SARS)-CoV-2 infection diagnosed in Italy, our country has rapidly taken its place within the rapidly evolving pandemic scenario. The increasingly restrictive measures adopted to contain SARS-CoV-2 infection and related Corona virus disease 2019 (Covid-19) have culminated into the national lockdown issued in March 2020. The inherent health emergency has inevitably put the Italian National Health Service to the test. Increasing the availability of beds dedicated to Covid-19 patients has reasonably become a high priority issue, which has imposed limits to the assistance provided to several non-Covid-19 patients, including cancer patients. In the present scenario, restrictions of those activities routinely intended as "non-deferrable", cannot be excluded. The postponement of interventions and procedures whose timing may impact treatment outcomes most commonly translates into prognostic worsening. To lower the risk of SARS-Cov-2 infection and Covid-19 in cancer patients, while guaranteeing continuity of care in this setting, each Italian Region has identified dedicated Covid-19 free hubs. The attempt to properly address these patients' needs has led to the establishment of treatment priorities even within this more restricted, still, extremely heterogeneous, group of patients. Therefore, guidelines informing the selection of non-deferrable cancer patients have been recently published. In specific referral to 
breast cancer patients, priorities have been identified for outpatient, diagnostic and surgical activities ${ }^{1,2}$. In patients undergoing breast surgery, surgical site infections (SSIs) occur at a frequency approximately varying within a 0.8 to $26 \%$ range $^{3-8}$. These latter events exert a negative impact on important patients' outcomes, including the timing of adjuvant treatment delivery and disease free survival. On this basis, and particularly in light of the current pandemic, actions taken to prevent the occurrence of SSIs in breast cancer patients undergoing non-deferrable breast surgery are of utmost importance. Indeed, if successfully applied, such actions may sensibly reduce the number of hospital accesses and the overall length of in hospital stay, which are both inevitably related to the risk of exposure to the SARS-Cov-2 and development of the inherent disease.

The Regina Elena National Cancer Institute (IRE) has been identified by the Lazio Region as a Covid-free hub. Consistently, measures have been applied in full accordance with the guidelines issued by the Italian Ministry of Health $(\mathrm{MoH})^{9}$.

It is reasonable foreseeing an impact of these same measures not only in terms of SARS-Cov-2 infection and disease, but also on the occurrence of SSIs in breast cancer patients undergoing surgery. We herein present data related to the experience matured at our Institute, i.e., a comprehensive cancer center certified by the Organization of European Cancer Centers (OECI), concerning the effects of the aforementioned restriction measures on the frequency and features of SSIs in breast cancer patients undergoing non-deferrable breast cancer surgery. The evidence presented will be discussed in reference to recent data exemplifying similar experiences from other National and International Institutions and discussed in light of the peculiar characteristics conferred by the Covid pandemic to the events observed.

\section{Patients And Methods}

\section{Study design}

We carried out an observational study according to a retrospective approach. The study protocol and the related informed consent form were approved by the local Ethical Review Board (protocol study number: 1382/20).

Our research primarily aimed at evaluating the impact of the extraordinary measures issued by the Italian $\mathrm{MoH}$ concerning the occurrence of infections at the surgical site in patients undergoing elective breast cancer surgery. To this purpose, two patients' cohorts were retrospectively identified and compared according to the methods reported below.

\section{Data collection}

The data herein analyzed and presented relate to breast cancer patients with an indication to non-deferrable breast surgery according to the Guidelines for Surgical Care released by the American College of Surgeons $(A C S)^{2}$ (Table 1). We also judged suitable for inclusion those breast cancer patients whose diagnosis had been made more than 1 month before. On this basis, the original table issued by the ACS has been enriched by this additional criterion, reported in the bottom row. Breast cancer patients who contributed data to our analysis were from 2 cohorts, namely, cohort 1, the lockdown cohort (LDC), and cohort 2, the non-lockdown cohort (NLDC). For patients from both these cohorts, a minimum 12-month follow up from breast surgery was required. Patients from the LDC underwent breast cancer surgery between the $15^{\text {th }}$ March 2020 and the $4^{\text {th }}$ May 2020 . Within this 
latter time frame, an ad hoc multi-disciplinary team oversaw the selection of breast cancer patients undergoing surgery according to the previously cited criteria. To this aim, weekly meetings were held. One single representative member for each of the following professional figures was involved: a general surgeon, oncologist, radiotherapist and plastic surgeon. Adequate social distance and mask wearing were constantly respected $^{10}$.

Patients from the LDC were compared with patients from the NLDC, i.e., patients having undergone nondeferrable breast cancer surgery and whose diagnosis had been made within the same time window postponed to the year 2019, i.e., between the15 ${ }^{\text {th }}$ March 2019 and $4^{\text {th }}$ May 2019. Patients were deemed not eligible and excluded if one or more among the following characteristic/s was/were represented: aged 80 years or older, diagnosed with autoimmune disorders, in course of therapy with steroids and/or within the past 6 months, American Society of Anesthesiologists (ASA) score equal to/greater than ( $\geq$ ) 4, body mass index (BMI) equal to greater than 35, kidney/liver/bone marrow failure. Ad hoc, pre-piloted forms were used for data retrieving from medical records. Data extraction was performed by specifically trained personnel and focused on demographic features, relevant clinical and pathological characteristics, ASA score, type and exact duration of surgery, drains, pre-surgical therapy/ies and co-morbidities.

For all the patients who contributed data to the present analysis, breast surgery was performed by the same team. In addition, postsurgical controls were performed regularly and, as already mentioned, for a minimum of 12-months. The interval length between consecutive controls varied mainly on the basis of breast implants positioning. Indeed, in the presence of implants, controls were set biweekly over the first three months and every two weeks for two additional months. Conversely, if no implants had been placed, controls were scheduled twice per week for a minimum of 30 days. A culture specimen was collected when an infected wound was suspected by the surgeon or in case of pain or tissue tenderness, swelling, redness or heat, purulent drainage or body temperature $>38^{\circ} \mathrm{C}$. SSIs were defined according to the criteria established by the Center for Disease Control and Prevention (CDC): purulent drainage (CDC\#1), a positive aseptically collected culture, (CDC\#2), at least one sign of inflammation with opening of the incision and absence of a negative culture (CDC\#3), or physician diagnosis of infection (CDC\#4) ${ }^{11}$.

The time elapsed between breast surgery and infection onset, prior therapy/ies (either medical or surgical), the need of re-hospitalization, and the impact on the postsurgical treatment administration were all recorded for each case of SSIs.

The present analysis does not include data from patients who were referred to other ambulatories following surgery or for whom the post-surgical controls were partially or totally unfeasible. The peri-operative measures routinely in place at our Surgical Division for the prevention of SSIs are fully consistent with the related recommendations from the World Health Organization (WHO) and have not changed over the last two years ${ }^{12}$ (Table 2). The measures adopted by the health care management team during the lockdown period in terms of limiting hospital accesses, sanitizing common areas such as ambulatories, waiting rooms and surgical rooms, interpersonal distancing, and use of individual protective devices are summarized in Table 3.

Statistical analysis 
Results were reported as means, medians, standard deviations and ranges if related to quantitative variables, while qualitative variables will be reported as absolute frequencies. Associations were estimated throughout chisquare or fisher exact test. For quantitative variables, groups were compared by Mann-Whitney test. All the analyses were performed using the SPSS 21.0 software (SPSS Inc., Chicago, Illinois, USA).

\section{Additional information}

\section{Ethical standards}

Informed consent was obtained from all patients participating in the study. The research was conducted according to the principles established by the Declaration of Helsinki.

\section{Results}

In the time window between the $15^{\text {th }}$ March 2020 and the 4th May 2020, at our Surgery Division, 79 breast cancer patients underwent breast surgery. Among them, 64 fulfilled the previously cited inclusion criteria. Subsequently, 2 patients out of these 64 were excluded, since having performed post-surgery controls at different Institutions. In the time frame between the $15^{\text {th }}$ March 2019 and the $4^{\text {th }}$ May 2019, 64 breast cancer patients underwent breast surgery at our Surgery Division. Of them, 54 fulfilled the preset inclusion criteria. Two of them were excluded due to incomplete data and post-surgery controls performed at different Institutions, respectively (Figure 1).

No relevant differences emerged when comparing the LDC and NLDC by descriptive statistics on demographic characteristics, main clinical-pathological features, ASA score, type and duration of surgery, use of drains, presurgical therapy/ies, and comorbidities (Table 4).

The impact of key patient-and breast surgery-related features on the outcome of interest, i.e., SSIs, as defined by the criteria established by the $\mathrm{CDC}$ is summarized in Table 5 . When globally considering the 114 breast cancer patients, from both the LDC and the NLDC, we observed significant evidence of lower SSIs in groups of patients having undergone skin reducing mastectomy compared with the remaining subgroups $(p=0.003)$.

The overall number of surgical site infections registered is $10(8.7 \%)$. The related distribution is as it follows: CDC\#1: 2 case, CDC\#2: 1 case, CDC\#3: 2 cases, and CDC\#4: 5 cases. The median to the occurrence of surgical site infection was 17.1 days, within 7 to 42 -day range.

Within the NLDC, the number of surgical site infections identified was 7 (13.5), with the related distribution being: CDC\#1: 2 cases, CDC\#2: 1 case, CDC\#3: 1 case, CDC\#4: 3 cases. The median time to the occurrence of surgical site infection was 18.2 days, within a 7 to 42-day range. Three patients were excluded, 2 CDC\#1 and 1

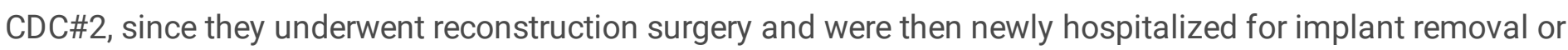
expander removal after 7, 11 and 15 days from the first breast surgery. In these latter cases, the short interval between the two consecutive surgeries did not delay the administration of the adjuvant treatment. The remaining four cases were successfully treated with medical therapy. In two out of four cases, adjuvant therapy was postponed till a 4-week delay was configured. In the LDC, 3 (4.8\%) surgical site infections occurred, with the following distribution: CDC\#3: 1 cases, and CDC\#4: 2 cases. The median time to the occurrence of surgical site 
infections was 14.3 days, within a 7 to 21 -day range. None of these patients was in need of re-hospitalization and no delay in the adjuvant treatment starting was recorded.

\section{Discussion}

Prevention of SSIs is a mainstay not only in breast surgery but more generally in oncologic surgery. Even though the breast and axillary wounds are classified as "clean," with an expected SSIs rate of less than $2 \%$, the overall incidence across different sources of statistics varies within and extremely wide range, i.e., from 1 to $30 \% 13$. SSIs may lead to prolonged hospital stay and, consequently, to increased costs. Additional related consequences may include poor cosmetic results, and higher chances of reconstruction failure. All of these may significantly contribute to patients' distress. Furthermore, surgical site infections, occasionally, may impact the oncologic care of cancer patients due to a delay in adjuvant therapies' administration and impaired postoperative surveillance, that potentially increases local and systemic cancer recurrence rates ${ }^{3,14}$. Patient- and procedure-related risk factors associated with higher rates of SSIs, along with potentially successful prevention strategies, have been widely described in literature. A high ASA score, old age, co-morbidities (e.g., diabetes, hypertension), smoking habit, prior surgery/ies, neoadjuvant therapy, use of drains therapy, major surgery, and prolonged surgery length are all independent risk factors and at least partly predictable risk factors of surgical site infections ${ }^{15}$. The prophylactic administration of antibiotics following breast cancer surgery is widely accepted $^{16}$.

Recently, as consequence of the rapid spread of Covid-19 in Italy, a sudden reorganization of the hospital structures was implemented by National and Regional Health System. The challenge for public health system was to reallocate resources and operators to face the virus emergency assuring at the same time resources to be made available for the emergency and for guaranteeing the continuity of standard care non-oncologic and oncologic patients. Initial evidence has suggested that patients with cancer may be more susceptible to infection and more likely to have higher surgery-related morbidity and mortality rates than the general population ${ }^{17}$. Thus, Covid-19 free cancer hubs were established in each Italian region in order to ensure safe and adequate treatment to non-Covid cancer patients. Indeed, also the activity of these cancer hubs were reconfigured. Patients were selected according the most recent recommendations and ad hoc internal operative procedures to minimize the virus diffusion were. In the Lazio Region, Central Italy, when the national lockdown was declared, the IRE of Rome was identified as a Covid-19 free cancer hub. At our Institute, our Unit of Breast Surgery is a high volume surgical center, which includes breast and plastic and reconstructive surgery. Specific internal protocols to preserve cancer patients from SARS-CoV-2 infection and disease have been adopted by the institutional risk management team, in full agreement with the guidelines released from the Italian Minister of Health and Regional Health Department. Our surgical activity during the Covid-19 pandemic has been described in detail elsewhere ${ }^{10}$. The work herein presented is aimed to highlight the effect of protective measures adopted in response to the Covid-19 pandemic on surgical site infections. To the best of our knowledge, limited evidence is available on the effects of such restriction measures on the occurrence of surgical site infections in breast cancer patients having undergone non-deferrable surgery.

Our results are consistent with the evidence made available by Intuitions similar to ours by patients flow and high standard of care. Overall, we observed a surgical site infection rate of $13.5 \%$ and $4.7 \%$ in patients from the NLDC and LDC, respectively. Lack of statistical significance between these two groups may reflect the restricted 
sample size, which surely represents an important limitation of our study from a methodological standpoint. We also found evidence of a significantly lower number of SSIs cases in patients who had performed skin reducing mastectomy. This data is consistent with the finding from Pechevy and collegues ${ }^{18}$. In addition, we noticed a qualitative difference between the two cohorts compared. Indeed, in the NLDC, 2 patients needed rehospitalization and implant/expander removal and 2 further patients were forced to prolonged medical therapy, with a consequent delay longer than 4 weeks in the administration of adjuvant treatment. Conversely, none of the breast cancer patients from the LDC required re-hospitalization and there were no delays in adjuvant therapy initiation. Since no updates have been recently provided for the prevention of SSIs and no significant differences emerged when comparing the two cohorts by relevant patient- and disease characteristics, it is our opinion that the measures adopted at the Institutional level in full accordance with the policy issued by the Italian $\mathrm{MoH}$ have contributed to lower the frequency of occurrence of SSIs in breast cancer patients undergoing breast surgery. Unfortunately, the quite restricted number of patients included in the two cohorts has importantly limited the statistic power of our analysis and does not allow for any firm conclusions. In addition, since the measures issued to limit the exposure of our patients to the SARS-Cov-2 vary within a quite wide range (Table 3 ), it is difficult to understand whether or not some of them have exerted a more relevant role in performing the supposed "lowering" action on the risk of exposure to the virus and disease development. In addition, when the lockdown period came to its end, some of preventive measures were modified or abolished and new protocols were issued. The analysis of data on SSIs rates under these revisited protocols and the comparison with similar data from the original protocols will probably provide more granular evidence on the differential impact of the actions taken on the outcomes of interest. Notwithstanding the previously cited limitations, some considerations may stem from the evidence herein described. Overall, the actions taken during the lockdown period seem to be cost-less or have affordable costs. On the other hand, when adopted, these restrictive measures seem to be associated with lower rates of surgical site infections in breast cancer patients. they seem to be effective in lowering the surgical. In addition, in an ad hoc questionnaire addressing the quality of care during the lockdown period, our patients seemed to consistently appreciate the quality of the assistance provided and were overall favorable to the adoptions of such restrictions. The containment measures were all perceived as "necessary and protective" and not "unnecessarily oppressive" by our patients. On this basis, we may hypothesize that most of these actions would be accepted on a stable basis by our patients if associated with a significant reduction in surgical site infections' rates.

\section{Conclusions}

Our data seem to support the role of the restrictive measures adopted during the lockdown period in lowering the rates of surgical site infections in breast cancer patients undergoing non-deferrable breast surgery. However, our results must also be interpreted in light of our study limitations, which are mainly represented by the quite restricted size of the cohorts compared. Similar evidence from other Covid-19 free hubs operating at the national level may help clarify our results.

However, based on the experience matured at our Institute, the adoption of the restrictive measures issued during the lockdown period has allowed to lower the chance of SARS-Cov-2 infection and disease in cancer patients and, at the same time, has maximized the chance for continuity of care at high quali-quantitative also during the lockdown period. 


\section{Abbreviations}

SARS-CoV-2: Severe acute respiratory syndrome coronavirus-2, Covid-19: Coronavirus disease 2019, SSIs:

surgical site infections, IRE: Regina Elena National Cancer Institute, MoH: Ministry of Health, OECl: Organization of European Cancer Centers, ACS: American College of Surgeons, LDC: lockdown cohort, NLDC: non- lockdown cohort, ASA: American Society of Anesthesiologists, BMI: body mass index, CDC: Center for Disease Control and Prevention, WHO: World Health Organization.

\section{Declarations}

Ethics approval and consent to participate

Approval was waived by the Institutional Review Board of the Regina Elena National Cancer Institute on the 21st July 2020 (Protocol\# RS N.1382/20). Informed consent was obtained from all patients participating in the study. This research was conducted according to the principles established by the Declaration of Helsinki.

Consent for publication

Not applicable.

Availability of data and materials

Data will be made from the corresponding author on reasonable request.

Competing interests

The authors declare no competing interests.

Funding

None.

Authors' contributions

SC, MC, CB: manuscript writing and conception, data collection and analysis. MB critical revision for important intellectual contents. DC, FP, FC study design. IS statistical analysis. PV, EC, LP, FG, LP: manuscript drafting. DM, $\mathrm{RDV}, \mathrm{VQ}, \mathrm{MP}, \mathrm{MC}, \mathrm{GC}$ data collection and critical revision.

The authors read and approved the final manuscript.

Acknowledgements

None.

Author details

${ }^{1}$ Department of Surgery, Division of Breast Surgery, IRCCS Regina Elena National Cancer Institute, Via Elio Chianesi 53, 00144-Rome, Italy. 
${ }^{2}$ Department of Applied Clinical Sciences and Biotechnology, University of L'Aquila, Via Vetoio, 67100-L’Aquila, Italy.

${ }^{3}$ Division of Medical Oncology 2, IRCCS Regina Elena National Cancer Institute, Via Elio Chianesi 53, 00144Rome, Italy.

${ }^{4}$ Biostatistics, IRCCS Regina Elena National Cancer Insitute, 00144-Rome, Italy.

${ }^{5}$ Department of Surgery, Division of Plastic and Reconstructive Surgery, IRCCS Regina Elena National Cancer Institute, Via Elio Chianesi 53, 00144- Rome, Italy.

${ }^{6}$ Scientific Direction, IRCCS Regina Elena National Cancer Institute, Via Elio Chianesi 53, 00144- Rome, Italy.

\section{References}

1. https://www.esmo.org/guidelines/cancer-patient-management-during-the-covid-19-pandemic/breastcancer-in-the-covid-19-era, last accessed on 23rd April 2021.

2. https://www.facs.org/media/files/covid19/guidance_for_triage_of_nonemergent_surgical_procedures.ashx, last accessed on 23rd April 2021.

3. Indelicato D, Grobmyer SR, Newlin H, et al. Association between operative closure type and acute infection, local recurrence, and disease surveillance in patients undergoing breast conserving therapy for early-stage breast cancer. Surgery. 2007,141(5):645-653. doi: 10.1016/j.surg.2006.12.011.

4. Prospero E, Cavicchi A, Bacelli S et al. Surveillance for surgical site infection after hospital discharge: a surgical procedure-specific perspective. Infect Control Hosp Epidemiol. 2006,27(12):1313-1317. doi:10.1086/509838

5. Edwards JR, Peterson KD, Mu Y, et al. National Healthcare Safety Network (NHSN) report: data summary for 2006 through 2008, issued December 2009. Am J Infect Control. 2009,37(10):783-805. doi: 10.1016/j.ajic.2009.10.001.

6. Nahabedian MY, Tsangaris T, Momen B, Manson PN. Infectious complications following breast reconstruction with expanders and implants. Plast Reconstr Surg. 2003,112(2):467-476. doi:10.1097/01.PRS.0000070727.02992.54-

7. Ruvalcaba-Limón E, Robles-Vidal C, Poitevin-Chacón A et al. Complications after breast cancer surgery in patients treated with concomitant preoperative chemoradiation: A case-control analysis. Breast Cancer Res Treat. 2006,95(2):147-152. doi:10.1007/s10549-005-9058-y

8. Vilar-Compte D, Jacquemin B, Robles-Vidal C, Volkow P. Surgical site infections in breast surgery: casecontrol study. World J Surg. 2004,28(3):242-246. doi:10.1007/s00268-003-7193-3.

9. Gruppo di Lavoro ISS Prevenzione e Controllo delle Infezioni COVID-19. Indicazioni ad interim per la sanificazione degli ambienti interni nel contesto sanitario e assistenziale per prevenire la trasmissione di SARS-CoV 2. Versione del 14 maggio 2020. Roma: Istituto Superiore di Sanità, 2020. (Rapporto ISS COVID19, n. 20/2020 Rev.).

10. Pelle F, Cappelli S, Graziano F, Piarulli L, Cavicchi F, Magagnano D, De Luca A, De Vita R, Pozzi M, Costantini M, Varanese A, Panimolle M, Gullo PP, Barba M, Vici P, Vizza E, Cognetti F, Sanguineti G, Saracca E, Ciliberto 
G, Botti C. Breast cancer surgery during the Covid-19 pandemic: a monocentre experience from the Regina Elena National Cancer Institute of Rome. J Exp Clin Cancer Res. 2020 Aug 27, 39:171. doi: 10.1186/s13046020-01683-y. PMCID: PMC7450921.

11. Degnim, A.C., Throckmorton, A.D., Boostrom, S.Y. et al. Surgical Site Infection after Breast Surgery: Impact of 2010 CDC Reporting Guidelines. Ann Surg Oncol 19, 4099-4103 (2012).

12. Allegranzi, Benedetta et al New WHO recommendations on preoperative measures for surgical site infection prevention: an evidence-based global perspective. The Lancet Infectious Diseases, Volume 16, Issue 12, e276 - e287.

13. Degnim AC, Throckmorton AD, Boostrom SY, et al. Surgical site infection after breast surgery: impact of 2010 CDC reporting guidelines. Ann Surg Oncol. 2012,19(13):4099-4103. doi:10.1245/s10434-012-2448-6.

14. Murthy BL, Thomson CS, Dodwell D, et al. Postoperative wound complications and systemic recurrence in breast cancer. Br J Cancer. 2007,97(9):1211-1217.

15. Vilar-Compte D, Rosales S, Hernandez-Mello N, Maafs E, Volkow P. Surveillance, control, and prevention of surgical site infections in breast cancer surgery: a 5-year experience. Am J Infect Control. 2009,37(8):674679).

16. Gallagher M, Jones DJ, Bell-Syer SV. Consensus Guideline on Preoperative Antibiotics and Surgical Site Infection in Breast Surgery- Official Statement- The American Society of breast Surgeonswww.breastsurgeons.org. Prophylactic antibiotics to prevent surgical site infection after breast cancer surgery. Cochrane Database Syst Rev. 201909 26, 9:CD005360).

17. Liang W, Guan W, Chen R et al. Cancer patients in SARS-CoV-2 infection: A nationwide analysis in China. Lancet Oncol 2020,21:335-337.

18. Pechevy L, Carloni R, Guerid S, Vincent PL, Toussoun G, Delay E. Skin-Reducing Mastectomy in Immediate Reconstruction: How to Limit Complications and Failures. Aesthet Surg J. 2017 Jun 1,37(6):665-677. doi: 10.1093/asj/sjw258. PMID: 28171481.

\section{Tables}

Table 1. Indication to non-deferrable breast surgery according to the Guidelines for Surgical Care from the American College of Surgeons*

\begin{tabular}{|ll|}
\hline$\cdot$ & Neo-adjuvant patients finishing treatment \\
\hline$\cdot$ & Clinical Stage T2 and/or N1 Tumors \\
\hline$\cdot \quad$ Triple negative or HER2 positive patients* \& \\
$\cdot \quad$ Discordant biopsies likely to be malignant \\
$\cdot \quad$ Excision of malignant recurrence \\
\hline Patients waiting for surgery more than 30 days after diagnosis \\
\hline
\end{tabular}


* https://www.facs.org/-/media/files/covid19/guidance_for_triage_of_nonemergent_surgical_procedures.ashx, reference 2

Table 2. Summary of measures implemented or initiated during the preoperative period and related WHO recommendations for the prevention of SISs*

\begin{tabular}{|ll|}
\hline$\cdot$ & Preoperative bathing \\
\hline$\cdot$ & Hair removal \\
\hline$\cdot$ & Optimal timing for administration of SAP \\
\hline$\cdot$ & Precise timing for administration of SAP \\
\hline$\cdot$ & Surgical hand preparation \\
\hline
\end{tabular}

WHO: World Health Organization SISs: surgical site infections; SAP: surgical antibiotics prophylaxis.

* Degnim, A.C., Throckmorton, A.D., Boostrom, S.Y. et al. Surgical Site Infection after Breast Surgery: Impact of 2010 CDC Reporting Guidelines. Ann Surg Oncol 2012; 19, 4099-4103 (Reference 11)

Table 3. Summary of measures taken by the health care management team during the lockdown period*

\begin{tabular}{|ll|}
\hline$\cdot$ & Major control and limitation of hospital access \\
\hline$\cdot$ & Personal protective equipment \\
\hline$\cdot$ & Standard disinfection \\
\hline$\cdot \quad$ & SPV disinfection of the operating room \\
\hline$\cdot$ & Self-monitoring of fever and respiratory symptoms by healthcare professionals \\
\hline
\end{tabular}

HPV: Hydrogen peroxide in the vaporized form

* Allegranzi, Benedetta et al. New WHO recommendations on preoperative measures for surgical site infection prevention: an evidence-based global perspective. The Lancet Infectious Diseases, Volume 16, Issue 12, e276 e287 (Reference 12)

Table 4. Patients' characteristics and surgical outcomes of the entire case-series (N: 114). 


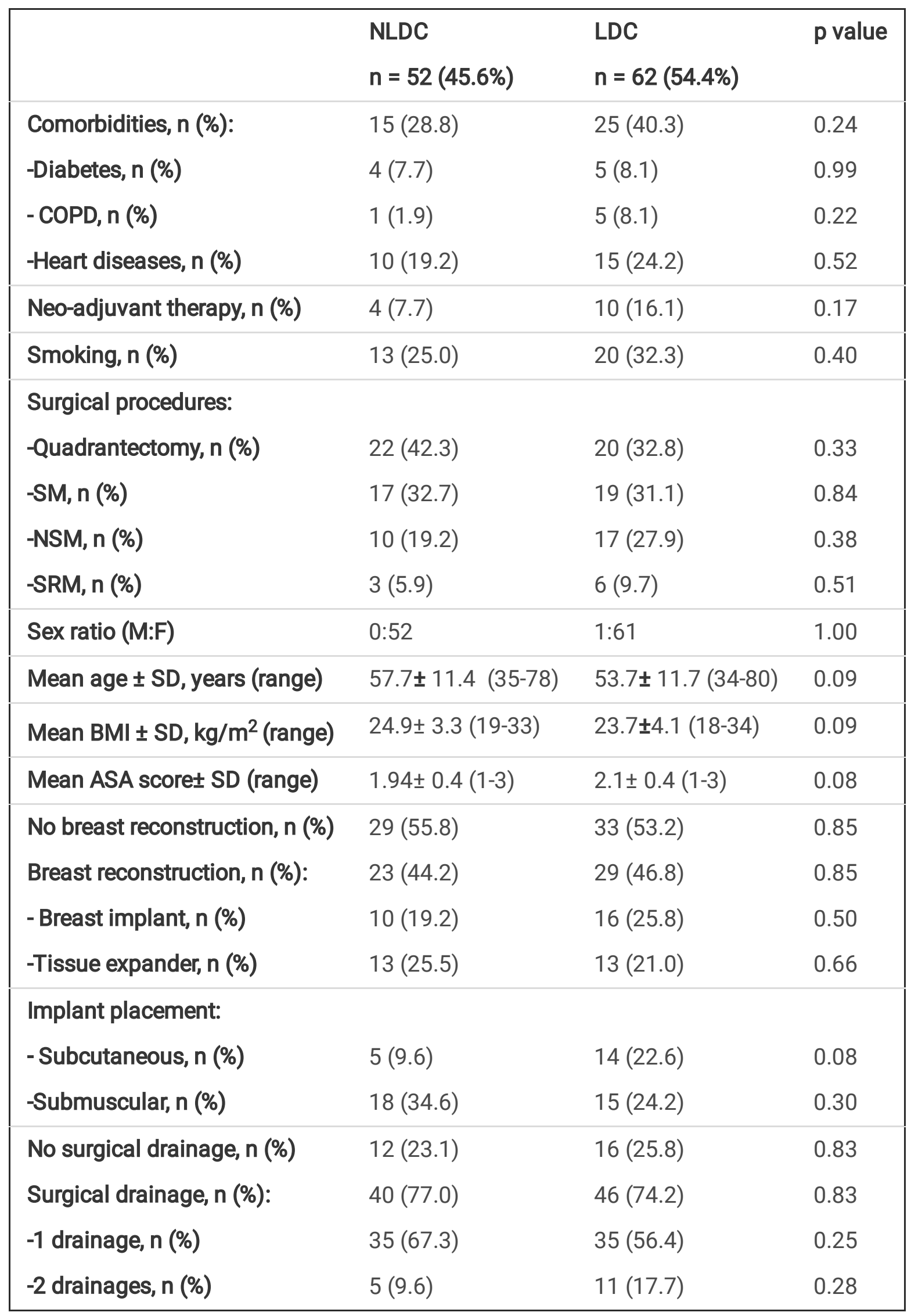

NLDC: no-lockdown cohort; LDC: lockdown cohort; COPD: chronic obstructive pulmonary disease; SM: simplex mastectomy; NSM: nipple sparing mastectomy; SRM: skin reducing mastectomy 
Table 5. The impact of key patient-and breast surgery-related features on the outcome of Surgical Site Infections as defined by the criteria established by the Centre for Disease Control (N: 114).

\begin{tabular}{|c|c|c|c|}
\hline & \multicolumn{2}{|c|}{ SSIs according to CDC criteria } & \multirow[t]{3}{*}{$\mathrm{p}$ value } \\
\hline & NO & YES & \\
\hline & $n=104(92.2 \%)$ & $n=10(8.8 \%)$ & \\
\hline COVID (No/Yes) & $45(43.3) / 59(56.7)$ & $7(66.7) / 3(33.3)$ & 0.182 \\
\hline *Comorbidities & $36(34.6)$ & $3(30)$ & 0.689 \\
\hline Smoking, n (\%) & $32(30.8)$ & $1(10.0)$ & 0.277 \\
\hline Mean age $\pm S D$, years (range) & $55.6 \pm 11.8(34-80)$ & $55.3 \pm 10.6(42-72)$ & 0.943 \\
\hline Mean $\mathrm{BMI} \pm \mathrm{SD}, \mathrm{kg} / \mathrm{m}^{2}$ (range) & $24.07 \pm 3.88(18-34)$ & $26.75 \pm 1.75(25-30)$ & 0.055 \\
\hline Mean ASA score \pm SD (range) & $2.02 \pm 0.39(1-3)$ & $2.13 \pm 0.35(2-3)$ & 0.113 \\
\hline Neo-adjuvant therapy, n (\%) & $13(12.5)$ & $1(10.0)$ & 1.000 \\
\hline \multicolumn{4}{|l|}{ Surgical procedures: } \\
\hline -Quadrantectomy, n (\%) & $40(38.5)$ & $2(20.0)$ & 0.320 \\
\hline -Simplex mastectomy, n (\%) & $33(31.7)$ & $3(30.0)$ & 1.000 \\
\hline -Nipple sparing mastectomy, n (\%) & $26(25.0)$ & $1(10.0)$ & 0.448 \\
\hline -Skin reducing mastectomy, n (\%) & $5(4.8)$ & $4(40.0)$ & 0.003 \\
\hline Breast reconstruction (No/Yes) & $57(54.8) / 47(45.2)$ & $5(50.0) / 5(50.0)$ & 1.000 \\
\hline Type of reconstruction: & & & 0.352 \\
\hline -Breast implant, n (\%) & $22(21.2)$ & $4(40.0)$ & \\
\hline - Tissue expander, n (\%) & $25(24.0)$ & $1(10.0)$ & \\
\hline \multicolumn{4}{|l|}{ Implant placement: } \\
\hline -Subcutaneous, n (\%) & $18(17.3)$ & $1(10.0)$ & 0.643 \\
\hline -Submuscular, n (\%) & $29(27.9)$ & $4(40.0)$ & \\
\hline Surgical drainage, $n$ (\%) & $77(74.0)$ & $6(60.0)$ & 0.424 \\
\hline
\end{tabular}

CDC: center for disease control; NLDC/LDC No-Lock Down Cohort/Lock Down Cohort *Included co-morbidities were one or more among the following: diabetes mellitus, chronic obstructive pulmonary disease and heart disease.

\section{Figures}



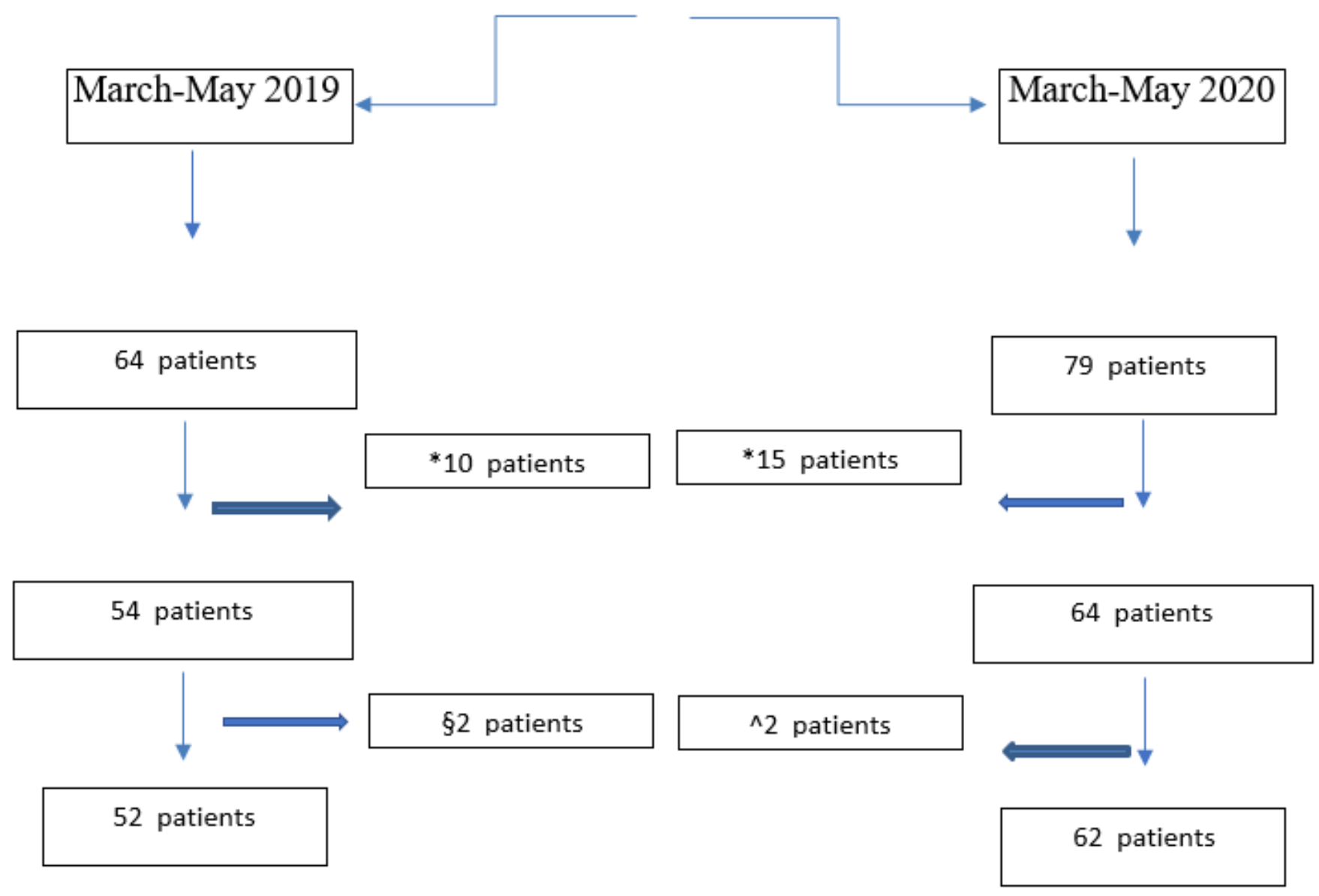

\section{Figure 1}

Study Flow Chart * These patients from each cohort were excluded because not fulfilling the inclusion criteria reported in table $1 \S$ These patients were lost to follow up ${ }^{\wedge}$ Of these 2 patients, 1 was excluded for incomplete data, and 1 was lost to follow up 